\title{
Complete Selection of a Self-Assembling Homo- or Hetero-Cavitand Cage via Metal Coordination Based on Ligand Tuning
}

Kenji Kobayashi, ${ }^{*}{ }^{\dagger}$ Yoshifumi Yamada, ${ }^{\dagger}$ Masamichi Yamanaka, ${ }^{\dagger}$ Yoshihisa Sei, ${ }^{\ddagger}, \S$ and Kentaro Yamaguchi",

${ }^{\dagger}$ Department of Chemistry, Faculty of Science, Shizuoka University, 836 Ohya, Shizuoka 422 8529, Japan.

${ }^{\ddagger}$ Chemical Analysis Center, Chiba University, Inage-ku, Chiba 263-8522, Japan.

${ }^{\S}$ Present address: Faculty of Pharmaceutical Sciences at Kagawa Campus, Tokushima Bunri University, Shido, Sanuki, Kagawa 769-2193, Japan.

E-mail: skkobay@ipc.shizuoka.ac.jp

\section{Supporting Information}

\section{Table of Contents}

- Synthetic procedures and spectral data of 1-3.

- ${ }^{1} \mathrm{H},{ }^{19} \mathrm{~F}$, and ${ }^{31} \mathrm{P}$ NMR spectral data of $\{2(\mathbf{2}) \bullet 4[\mathrm{Pd}(\mathrm{dppp})]\}^{8+} \bullet 8\left(\mathrm{TfO}^{-}\right)(\mathbf{5})$, $\{2(\mathbf{3}) \bullet 4[\mathrm{Pd}(\mathrm{dppp})]\}^{8+} \bullet 8\left(\mathrm{TfO}^{-}\right)(\mathbf{6})$, and $\{\mathbf{1} \bullet \mathbf{3} 4[\mathrm{Pd}(\mathrm{dppp})]\}^{8+} \bullet 8\left(\mathrm{TfO}^{-}\right)(\mathbf{7})$.

- Figure S1. Self-assembly of $\{2(2) \bullet 4[\mathrm{Pd}(\mathrm{dppp})]\}^{8+} \bullet 8\left(\mathrm{TfO}^{-}\right)(\mathbf{5})$ in the different molar ratios of 2 and Pd(dppp)(OTf) 2 (4) monitored by ${ }^{1} \mathrm{H}$ NMR.

- Figure S2. Self-assembly of $\mathbf{5}$ in the different molar ratios of $\mathbf{2}$ and $\mathbf{4}$ monitored by ${ }^{31} \mathrm{P}$ NMR.

- Figure S3. Self-assembly of $\{\mathbf{1} \cdot 3 \cdot 4[\mathrm{Pd}(\mathrm{dppp})]\}^{8+} \bullet 8\left(\mathrm{TfO}^{-}\right)(\mathbf{7})$ in the different molar ratios of 1, 3, and 4 monitored by ${ }^{1} \mathrm{H}$ NMR.

- Figure S4. ${ }^{31} \mathrm{P}$ NMR spectrum of 7.

- Figure S5. CSI-MS spectra of 5 and 7. 
General. ${ }^{1} \mathrm{H}$ and ${ }^{13} \mathrm{C}$ NMR spectra were recorded at $300 \mathrm{MHz}$ and $75 \mathrm{MHz}$, respectively, on a Bruker AC300 spectrometer. ${ }^{19} \mathrm{~F}$ and ${ }^{31} \mathrm{P}$ NMR spectra were recorded at $376 \mathrm{MHz}$ and $162 \mathrm{MHz}$, respectively, on a JEOL JNM-AL400 spectrometer. IR spectra were obtained on a JASCO FT/IR-460Plus spectrometer. CSI-MS spectra were measured on a JEOL JMS-700 spectrometer. Tetraiodocavitand $\left(\mathrm{R}=\left(\mathrm{CH}_{2}\right)_{6} \mathrm{CH}_{3}\right)$ was synthesized according to the literature. ${ }^{1}$

Tetra(4-pyridyl)-cavitand (1). To a mixture of tetraiodocavitand (716.4 mg, 0.500 $\mathrm{mmol}), \mathrm{PdCl}_{2}\left(\mathrm{PPh}_{3}\right)_{2}(87.7 \mathrm{mg}, 0.125 \mathrm{mmol})$, and $\mathrm{Cs}_{2} \mathrm{CO}_{3}(2.44 \mathrm{~g}, 7.49 \mathrm{mmol})$ in 1,4-dioxane $(25 \mathrm{~mL})$ and $\mathrm{H}_{2} \mathrm{O}(2 \mathrm{~mL})$ under $\mathrm{Ar}$ was added a solution of 4-(4,4,5,5-tetramethyl-1,3,2dioxaborolan-2-yl)pyridine (1.025 g, $5.00 \mathrm{mmol})$ and $\mathrm{AsPh}_{3}(306.2 \mathrm{mg}, 1.00 \mathrm{mmol})$ in 1,4dioxane $(30 \mathrm{~mL})$. The resulting mixture was stirred at $110{ }^{\circ} \mathrm{C}$ for $30 \mathrm{~h}$ under Ar. After cooling to room temperature and then adding $\mathrm{CHCl}_{3}$, the reaction mixture was filtered. The filtrate was washed with $\mathrm{H}_{2} \mathrm{O}$ and brine and dried over $\mathrm{Na}_{2} \mathrm{SO}_{4}$. After evaporation of solvents, the residue was purified with column chromatography on silica gel eluted with EtOAc-EtOH (1:4) containing $3 \% \mathrm{Et}_{3} \mathrm{~N}$ followed by reprecipitation with $\mathrm{CHCl}_{3}$-hexane to give 1 (569.7 $\mathrm{mg}$, $92 \%$ yield) as a white solid. $\mathrm{Mp} 231{ }^{\circ} \mathrm{C} ;{ }^{1} \mathrm{H} \mathrm{NMR}\left(\mathrm{CDCl}_{3}\right) \delta 0.92(\mathrm{t}, J=6.8 \mathrm{~Hz}, 12 \mathrm{H}), 1.25-$ $1.58(\mathrm{~m}, 40 \mathrm{H}), 2.28-2.42(\mathrm{~m}, 8 \mathrm{H}), 4.22(\mathrm{~d}, J=6.9 \mathrm{~Hz}, 4 \mathrm{H}), 4.85(\mathrm{t}, J=7.8 \mathrm{~Hz}, 4 \mathrm{H}), 5.30(\mathrm{~d}, J$ $=6.9 \mathrm{~Hz}, 4 \mathrm{H}), 6.98(\mathrm{~d}, J=5.8 \mathrm{~Hz}, 8 \mathrm{H}), 7.37(\mathrm{~s}, 4 \mathrm{H}), 8.61(\mathrm{~d}, J=5.8 \mathrm{~Hz}, 8 \mathrm{H}) ;{ }^{13} \mathrm{C} \mathrm{NMR}$ $\left(\mathrm{CDCl}_{3}\right) \delta 14.1,22.6,27.9,29.3,29.7,30.3,31.6,36.9,100.4,120.9,124.9,126.8,138.6$, 142.1, 149.3, 152.2; IR (KBr) v 2927, 1604, 1459, 1160, 1087, 1017, $966 \mathrm{~cm}^{-1}$. Anal. Calcd for $\mathrm{C}_{80} \mathrm{H}_{92} \mathrm{~N}_{4} \mathrm{O}_{8} \cdot \mathrm{H}_{2} \mathrm{O}: \mathrm{C}, 76.52 ; \mathrm{H}, 7.55 ; \mathrm{N}, 4.46$. Found: C, 76.39; H, 7.46; N, 4.43.

Tetrakis(4-pyridylethynyl)-cavitand (2). To a mixture of tetraiodocavitand (167.6 mg, $0.117 \mathrm{mmol}$ ), 4-ethynylpyridine (145.9 mg, $1.41 \mathrm{mmol}), \mathrm{PdCl}_{2}\left(\mathrm{PPh}_{3}\right)_{2}(12.4 \mathrm{mg}, 0.0177$ $\mathrm{mmol}), \mathrm{CuI}(4.5 \mathrm{mg}, 0.023 \mathrm{mmol})$, and $\mathrm{PPh}_{3}(6.1 \mathrm{mg}, 0.023 \mathrm{mmol})$ under Ar were added dry THF $(15 \mathrm{~mL})$ and dry $i$ - $\mathrm{Pr}_{2} \mathrm{NH}(15 \mathrm{~mL})$. The resulting mixture was stirred at $50{ }^{\circ} \mathrm{C}$ for $72 \mathrm{~h}$ under Ar. After evaporation of solvents, the residue was partitioned between $\mathrm{CHCl}_{3}$ and $\mathrm{H}_{2} \mathrm{O}$. The organic layer was washed with $\mathrm{H}_{2} \mathrm{O}$ and brine and dried over $\mathrm{Na}_{2} \mathrm{SO}_{4}$. After evaporation of solvent, the residue was purified with column chromatography on silica gel eluted with EtOAc-EtOH (2:1) containing $1 \% \mathrm{Et}_{3} \mathrm{~N}$ followed by reprecipitation with $\mathrm{CHCl}_{3}$-hexane to give $2\left(113.0 \mathrm{mg}, 72 \%\right.$ yield) as a white solid. $\mathrm{Mp} 241{ }^{\circ} \mathrm{C} ;{ }^{1} \mathrm{H} \mathrm{NMR}\left(\mathrm{CDCl}_{3}\right) \delta 0.92(\mathrm{t}, J=6.7$ $\mathrm{Hz}, 12 \mathrm{H}), 1.22-1.56(\mathrm{~m}, 40 \mathrm{H}), 2.25-2.32(\mathrm{~m}, 8 \mathrm{H}), 4.59(\mathrm{~d}, J=7.2 \mathrm{~Hz}, 4 \mathrm{H}), 4.87(\mathrm{t}, J=7.9$ $\mathrm{Hz}, 4 \mathrm{H}), 6.02(\mathrm{~d}, J=7.2 \mathrm{~Hz}, 4 \mathrm{H}), 7.17(\mathrm{~s}, 4 \mathrm{H}), 7.31(\mathrm{~d}, J=5.7 \mathrm{~Hz}, 8 \mathrm{H}), 8.61(\mathrm{~d}, J=5.7 \mathrm{~Hz}$, $8 \mathrm{H}) ;{ }^{13} \mathrm{C} \mathrm{NMR}\left(\mathrm{CDCl}_{3}\right) \delta 14.0,22.6,27.7,29.3,29.5,29.6,31.8,36.4,85.5,94.6,98.4,112.3$, 121.2, 125.5, 130.9, 138.6, 149.9, 155.6; IR (KBr) v 2926, 2214, 1594, 1449, 1151, 1092 , 
1019, $973 \mathrm{~cm}^{-1}$. Anal. Calcd for $\mathrm{C}_{88} \mathrm{H}_{92} \mathrm{~N}_{4} \mathrm{O}_{8}: \mathrm{C}, 79.25 ; \mathrm{H}, 6.95 ; \mathrm{N}, 4.20$. Found: $\mathrm{C}, 78.91 ; \mathrm{H}$, $6.95 ; \mathrm{N}, 4.21$.

Tetrakis(4-cyanophenyl)-cavitand (3). According to the same reaction and workup conditions for the preparation of $\mathbf{1}$, except for the use of 4-(4,4,5,5-tetramethyl-1,3,2dioxaborolan-2-yl)benzonitrile, 3 was obtained as a white solid in $89 \%$ yield. Purification: column chromatography on silica gel eluted with $\mathrm{CHCl}_{3}$-EtOAc $(50: 1)$ followed by reprecipitation with $\mathrm{CHCl}_{3}$-hexane. $\mathrm{Mp} 330{ }^{\circ} \mathrm{C} ;{ }^{1} \mathrm{H} \mathrm{NMR}\left(\mathrm{CDCl}_{3}\right) \delta 0.92(\mathrm{t}, J=6.9 \mathrm{~Hz}, 12 \mathrm{H})$, 1.24-1.58 (m, 40H), 2.26-2.46 (m, 8H), 4.18 (d, $J=7.0 \mathrm{~Hz}, 4 \mathrm{H}), 4.83(\mathrm{t}, J=8.0 \mathrm{~Hz}, 4 \mathrm{H}), 5.25$ $(\mathrm{d}, J=7.0 \mathrm{~Hz}, 4 \mathrm{H}), 7.14(\mathrm{~d}, J=8.2 \mathrm{~Hz}, 8 \mathrm{H}), 7.37(\mathrm{~s}, 4 \mathrm{H}), 7.65(\mathrm{~d}, J=8.2 \mathrm{~Hz}, 8 \mathrm{H}) ;{ }^{13} \mathrm{C} \mathrm{NMR}$ $\left(\mathrm{CDCl}_{3}\right) \delta 14.1,22.6,27.9,29.4,29.7,30.3,31.8,37.0,100.4,111.3,118.5,120.9,127.7$, 130.8, 131.7, 138.6, 138.8, 155.2; IR (KBr) v 2927, 2228, 1609, 1465, 1158, 1085, 1019, 967 $\mathrm{cm}^{-1}$. Anal. Calcd for $\mathrm{C}_{88} \mathrm{H}_{92} \mathrm{~N}_{4} \mathrm{O}_{8}: \mathrm{C}, 79.25 ; \mathrm{H}, 6.95 ; \mathrm{N}, 4.20$. Found: C, 78.98; H, 6.88; N, 4.20.

\section{Reference}

1) Barrett, E. S.; Irwin, J. L.; Turner, P.; Sherburn, M. S. J. Org. Chem. 2001, 66, 8227-8229. 
$\{2(2) \bullet 4[\operatorname{Pd}(\mathbf{d p p p})]\}^{8+} \bullet 8\left(\mathbf{T f O}^{-}\right)(\mathbf{5}) .{ }^{1} \mathrm{H} \mathrm{NMR}\left(\mathrm{CDCl}_{3}, 1 \mathrm{mM}, 23{ }^{\circ} \mathrm{C}\right) \delta 0.89(\mathrm{t}, J=6.7 \mathrm{~Hz}$, $24 \mathrm{H}), 1.19-1.51$ (m, 80H), 2.10-2.40 (m, 24H), 3.12-3.32 (m, 16H), 4.25 (d, J = 7.3 Hz, 8H), $4.76(\mathrm{t}, J=8.0 \mathrm{~Hz}, 8 \mathrm{H}), 5.92(\mathrm{~d}, J=7.3 \mathrm{~Hz}, 8 \mathrm{H}), 7.01$ (d, $J=6.3 \mathrm{~Hz}, 16 \mathrm{H}), 7.12(\mathrm{~s}, 8 \mathrm{H})$, 7.32-7.52 (m, 48H), 7.52-7.69 (m, 32H), $8.93(\mathrm{~d}, J=6.3 \mathrm{~Hz}, 16 \mathrm{H}) ;{ }^{19} \mathrm{~F} \mathrm{NMR}\left(\mathrm{CDCl}_{3}, 2 \mathrm{mM}\right.$, $\left.23{ }^{\circ} \mathrm{C}\right) \delta-79.91 ;{ }^{31} \mathrm{P}$ NMR $\left(\mathrm{CDCl}_{3}, 2 \mathrm{mM}, 23{ }^{\circ} \mathrm{C}\right) \delta 6.39(\mathrm{~s})$.
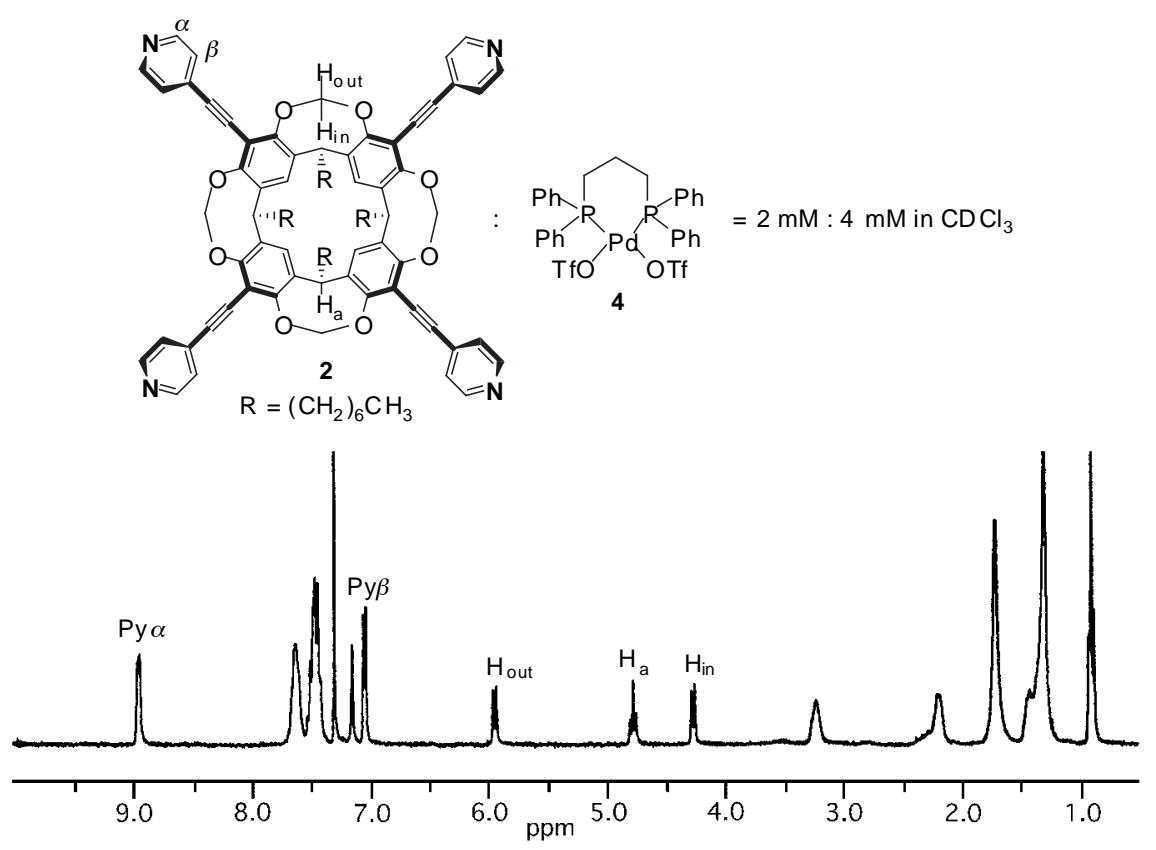

$\{2(3) \bullet 4[\mathbf{P d}(\mathbf{d p p p})]\}^{8+} \bullet 8\left(\mathbf{T f O}^{-}\right)(\mathbf{6}) .{ }^{1} \mathrm{H} \mathrm{NMR}\left(\mathrm{CDCl}_{3}, 1 \mathrm{mM}, 23{ }^{\circ} \mathrm{C}\right) \delta 0.91(\mathrm{t}, J=6.7 \mathrm{~Hz}$, $24 \mathrm{H}), 1.23-1.55(\mathrm{~m}, 80 \mathrm{H}), 2.22-2.47(\mathrm{~m}, 24 \mathrm{H}), 2.83-3.02(\mathrm{~m}, 16 \mathrm{H}), 4.27$ (d, J= 7.1 Hz, 8H), $4.74(\mathrm{t}, J=8.0 \mathrm{~Hz}, 8 \mathrm{H}), 4.94(\mathrm{~d}, J=7.1 \mathrm{~Hz}, 8 \mathrm{H}), 7.24(\mathrm{~d}, J=8.0 \mathrm{~Hz}, 16 \mathrm{H}), 7.31(\mathrm{~s}, 8 \mathrm{H})$, 7.38-7.53 (m, 64H (including 7.40, d, $J=8.0 \mathrm{~Hz}, 16 \mathrm{H})$ ), $7.70(\mathrm{dd}, J=8.0$ and $11.9 \mathrm{~Hz}, 32 \mathrm{H})$; ${ }^{19} \mathrm{~F} \mathrm{NMR}\left(\mathrm{CDCl}_{3}, 1 \mathrm{mM}, 23{ }^{\circ} \mathrm{C}\right) \delta-80.04 ;{ }^{31} \mathrm{P} \mathrm{NMR}\left(\mathrm{CDCl}_{3}, 1 \mathrm{mM}, 23{ }^{\circ} \mathrm{C}\right) \delta 13.34$ (brs).
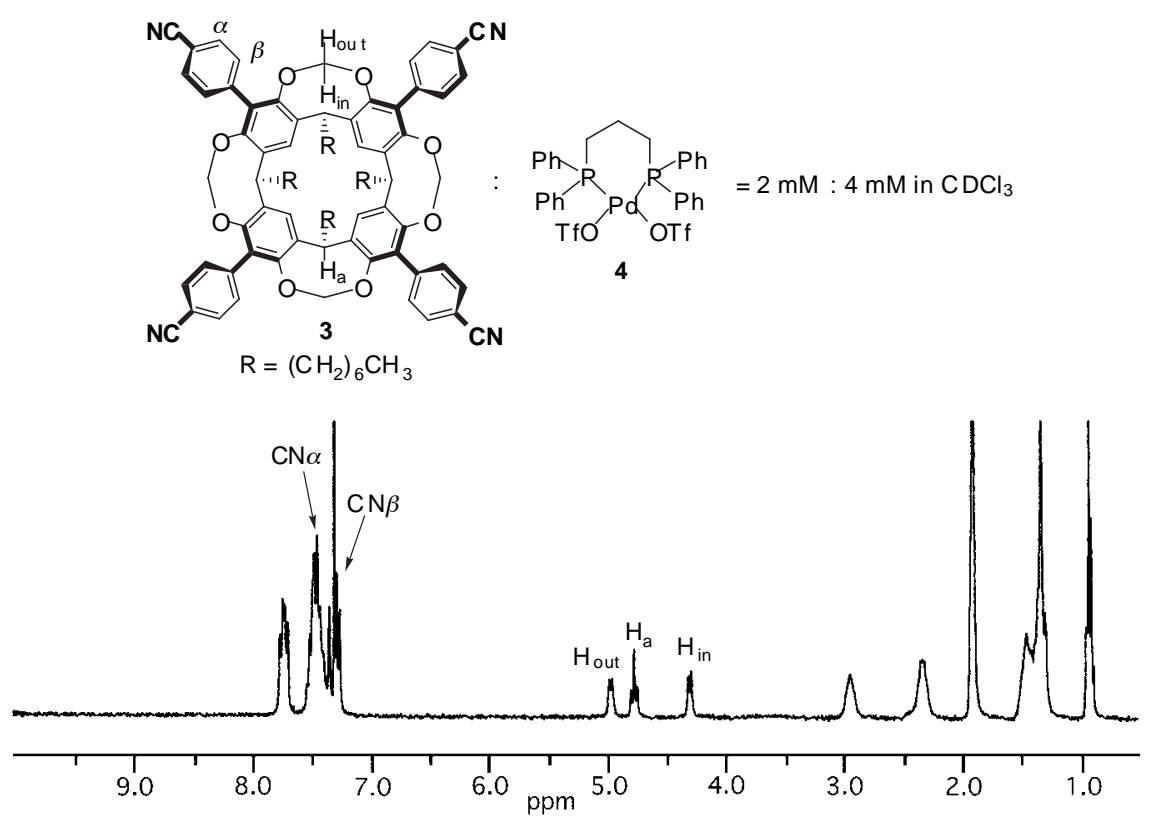
$\{1 \bullet 3 \bullet 4[\operatorname{Pd}(\mathbf{d p p p})]\}^{8+\bullet} 8\left(\mathbf{T f O}^{-}\right)(\mathbf{7}) .{ }^{1} \mathrm{H} \mathrm{NMR}\left(\mathrm{CDCl}_{3}, 1 \mathrm{mM}, 23{ }^{\circ} \mathrm{C}\right) \delta 0.76-0.94(\mathrm{~m}, 24 \mathrm{H})$, $1.12-1.54(\mathrm{~m}, 80 \mathrm{H}), 2.04-2.52(\mathrm{~m}, 24 \mathrm{H}), 2.73-3.01(\mathrm{~m}, 8 \mathrm{H}), 3.17-3.87(\mathrm{~m}, 8 \mathrm{H}), 4.23(\mathrm{~d}, J=$ $6.4 \mathrm{~Hz}, 4 \mathrm{H}, 3-\mathrm{OCH} i n), 4.38$ (d, J = 6.6 Hz, 1-OCHin), 4.70 (t, $J=7.9 \mathrm{~Hz}, 4 \mathrm{H}, 1$-methyne), 4.83 (t, $J=8.2 \mathrm{~Hz}, 4 \mathrm{H}, 3$-methyne), 4.85 (d, $J=6.4 \mathrm{~Hz}, 4 \mathrm{H}, 3-\mathrm{OCH}$ out), 5.62 (d, $J=6.6 \mathrm{~Hz}$, 4H, 1-OCHout), 6.98 (d, $J=5.9$ Hz, 8H, 1-Py $\beta H), 7.02-7.10(\mathrm{~m}, 8 \mathrm{H}), 7.10-7.21$ (m, 28H (including 7.19, brs, 8H, 3-PhCN $\beta H)$ ), 7.30-7.48 (m, 20H), 7.48-7.62 (m, 32H (including 7.56, brs, $8 \mathrm{H}, 3-\mathrm{PhCN} \alpha H)$ ), 7.70-7.90 (m, 16H), 8.90 (brs, 8H, 1-Py $\alpha H) ;{ }^{19} \mathrm{~F} \mathrm{NMR}\left(\mathrm{CDCl}_{3}, 2\right.$ $\left.\mathrm{mM}, 23{ }^{\circ} \mathrm{C}\right) \delta-80.03 ;{ }^{31} \mathrm{P} \mathrm{NMR}\left(\mathrm{CDCl}_{3}, 2 \mathrm{mM}, 23{ }^{\circ} \mathrm{C}\right) \delta 6.39\left(\mathrm{~d},{ }^{3} J_{\mathrm{pp}}=27.3 \mathrm{~Hz}\right), 9.51\left(\mathrm{~d},{ }^{3} J_{\mathrm{pp}}\right.$ $=27.3 \mathrm{~Hz})$.
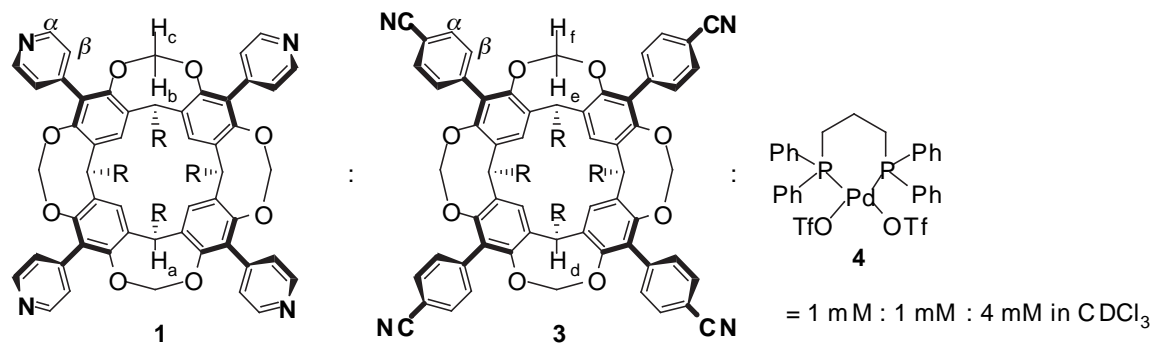

$\mathrm{R}=\left(\mathrm{CH}_{2}\right)_{6} \mathrm{CH}_{3}$

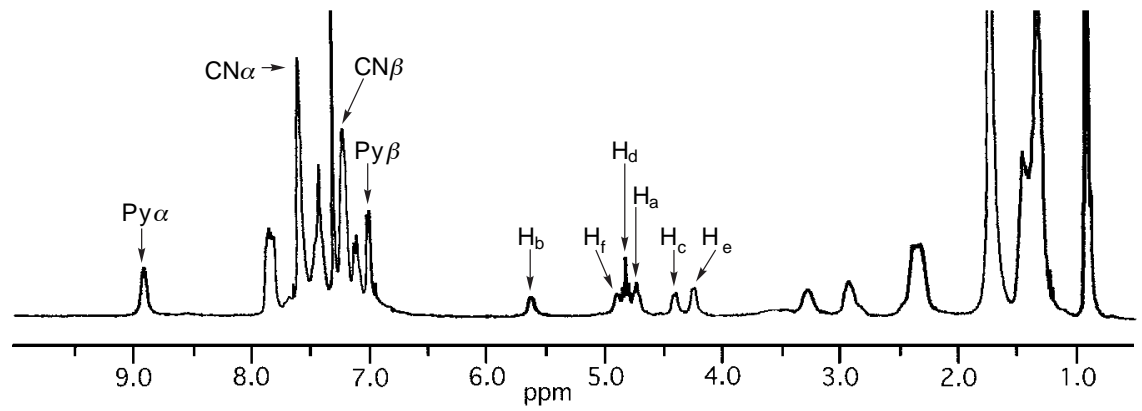




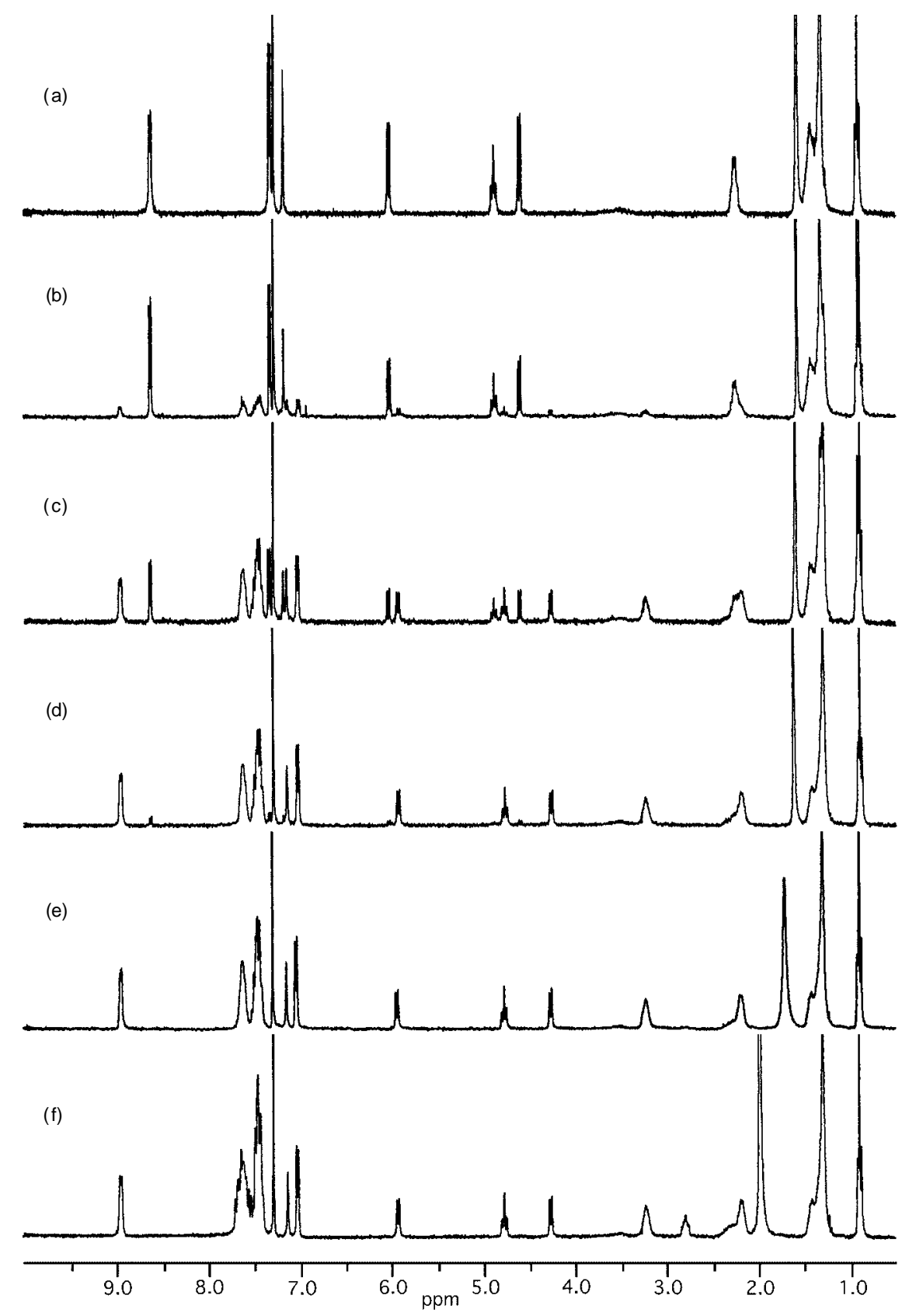

Figure S1. Self-assembly of $\{2(2) \bullet 4[\mathrm{Pd}(\mathrm{dppp})]\}^{8+} \cdot 8\left(\mathrm{TfO}^{-}\right)(\mathbf{5})$ in the different molar ratios of 2 and 4. ${ }^{1} \mathrm{H}$ NMR spectra of a mixture of $2(2 \mathrm{mM})$ and 4 in $\mathrm{CDCl}_{3}$ at $23{ }^{\circ} \mathrm{C}$ in the molar ratios of (a) 2:0, (b) 2:1, (c) 2:2, (d) 2:3, (e) 2:4, and (f) 2:8. 

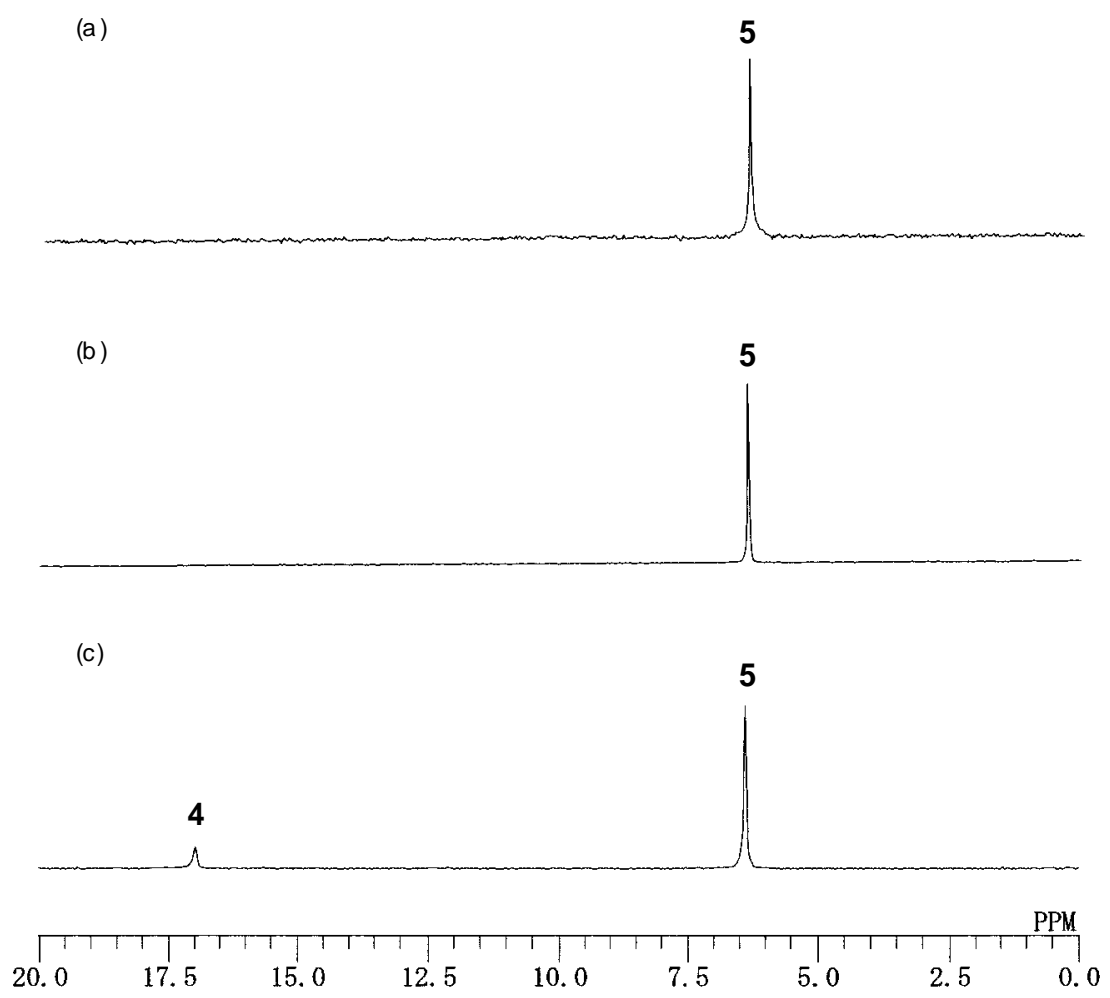

Figure S2. Self-assembly of 5 in the different molar ratios of 2 and $4 .{ }^{31} \mathrm{P}$ NMR spectra of a mixture of 2 (4 mM) and 4 in $\mathrm{CDCl}_{3}$ at $23{ }^{\circ} \mathrm{C}$ in the molar ratios of (a) 2:2, (b) 2:4, and (c) 2:6. 


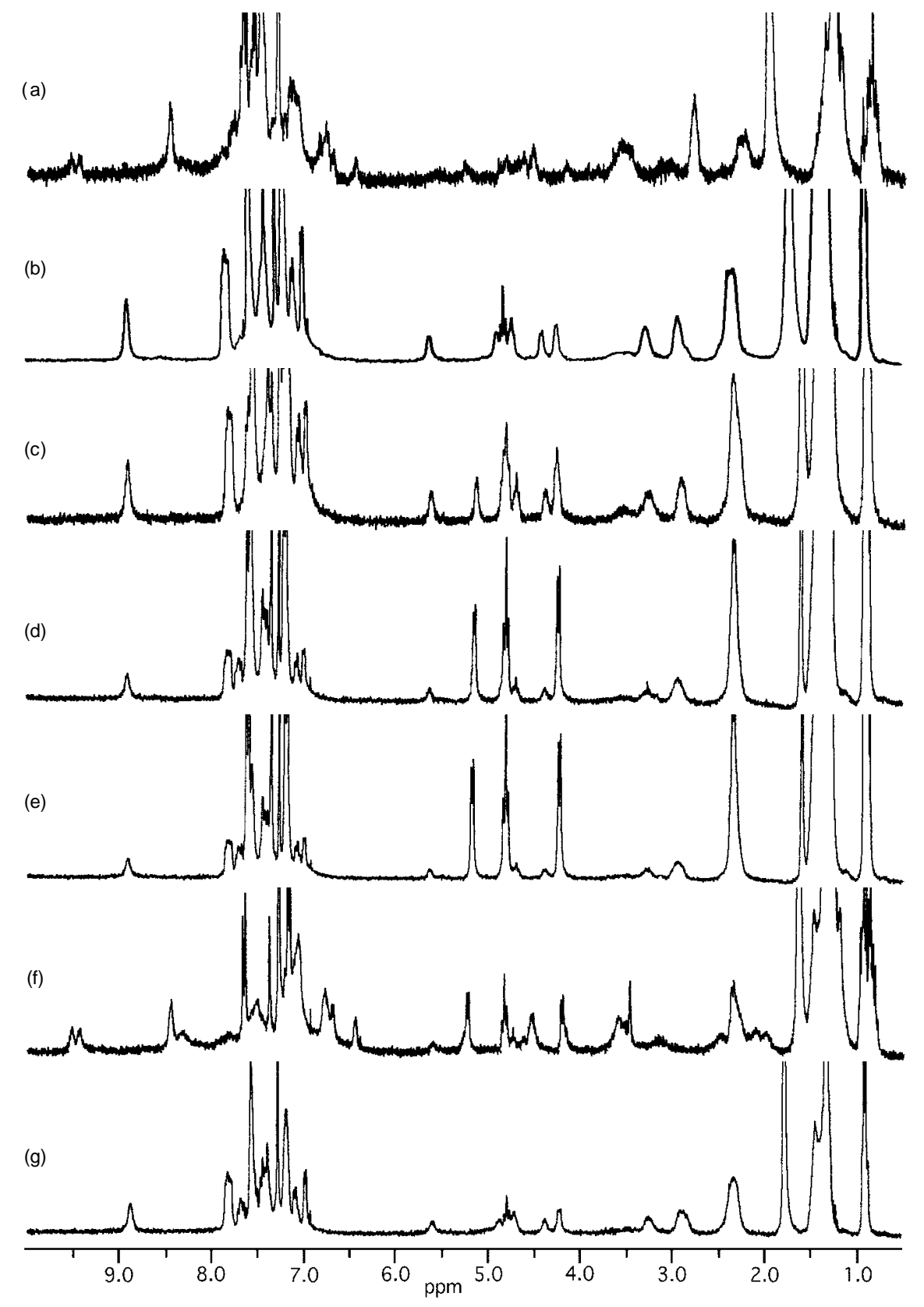

Figure S3. Self-assembly of $\{\mathbf{1} \cdot \mathbf{3} \cdot 4[\mathrm{Pd}(\mathrm{dppp})]\}^{8+} \bullet 8\left(\mathrm{TfO}^{-}\right)(\mathbf{7})$ in the different molar ratios of $\mathbf{1 , 3}$, and 4. ${ }^{1} \mathrm{H}$ NMR spectra of a mixture of $\mathbf{1 , 3}$, and $4(4 \mathrm{mM})$ in $\mathrm{CDCl}_{3}$ at $23{ }^{\circ} \mathrm{C}$ in the molar ratios of (a) 1:0:4, (b) 1:1:4, (c) 1:2:4, (d) 1:4:4, (e) 1:6:4, (f) 2:1:4, and (g) after addition of 1 equiv of $\mathbf{3}$ to the sample (a). 


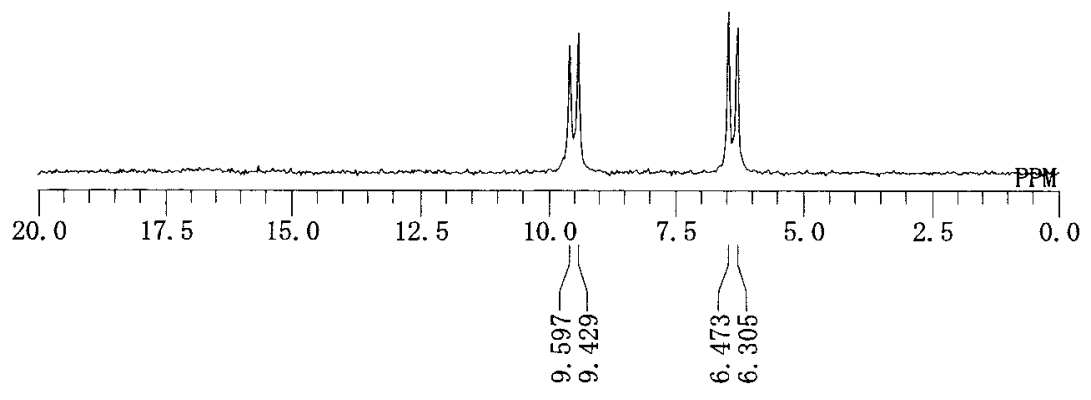

Figure S4. ${ }^{31} \mathrm{P}$ NMR spectrum of $7(2 \mathrm{mM})$ in $\mathrm{CDCl}_{3}$ at $23{ }^{\circ} \mathrm{C}$.

(a) $[2(2) \cdot 4\{\mathrm{Pd}(\mathrm{dppp})\}]^{8+} \bullet 8 \mathrm{TfO}-$

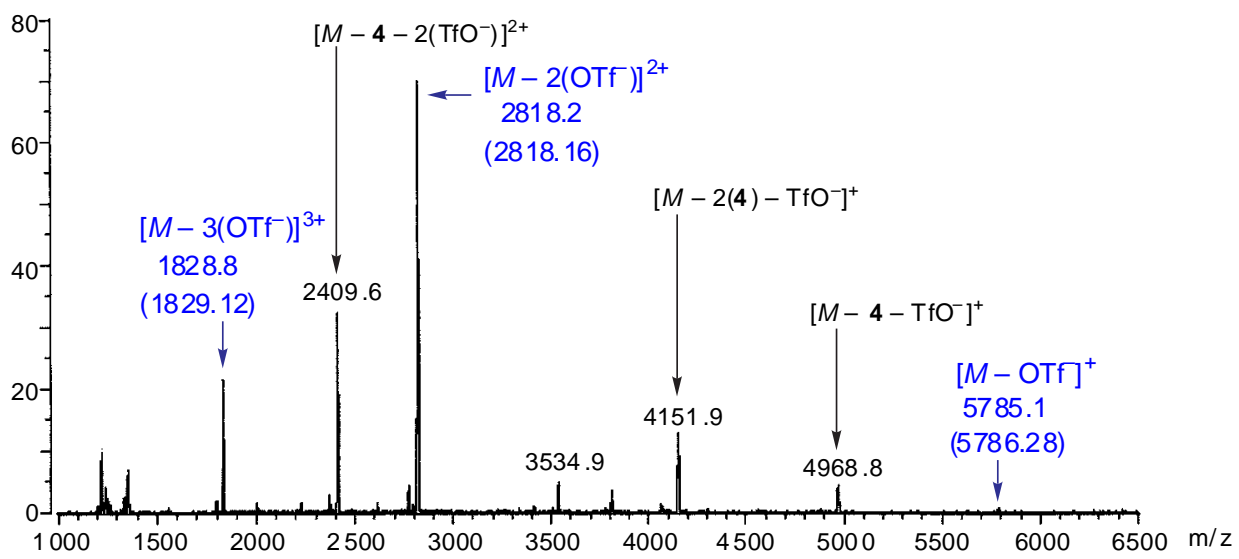

(b) $[1 \cdot 3 \cdot 4\{\mathrm{Pd}(\mathrm{dppp})\}]^{8+} \cdot 8 \mathrm{TfO}-$

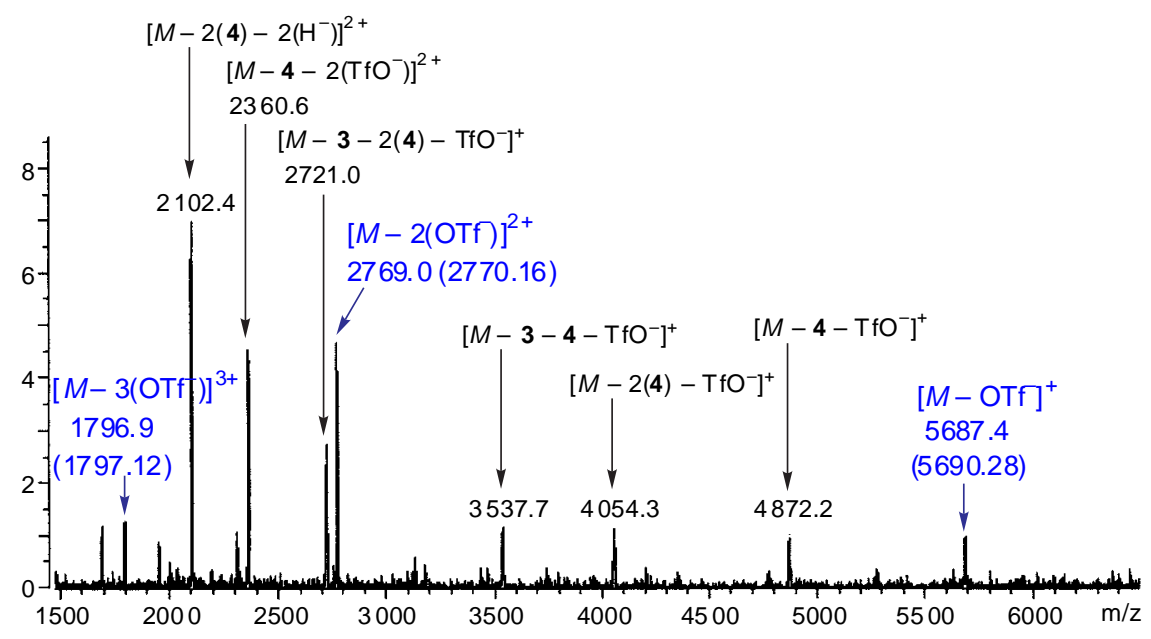

Figure S5. CSI-MS spectra (spray temperature $=-20{ }^{\circ} \mathrm{C}$; ion source temperature $=25^{\circ} \mathrm{C}$ ): (a) $[2]=1 \mathrm{mM}$ and $[4]=2 \mathrm{mM}$ in $\mathrm{CHCl}_{3}$; and (b) $[\mathbf{1}]=[3]=0.5 \mathrm{mM}$ and $[4]=2 \mathrm{mM}$ in $\mathrm{CHCl}_{3}$. 